\title{
Concepción frente al consumo de alcohol en jóvenes de la Universidad del Cauca
}

\author{
Conception to alcohol consumption in young people from the University of Cauca
}

Nancy Janneth Molano-Tobar ${ }^{1}$, Dolly Ximena Molano-Tobar ${ }^{*}$

\begin{abstract}
1 Fisioterapeuta, Magister en Educación con énfasis en Fisiología del Deporte. Docente titular Universidad del Cauca. Popayán, Colombia.e-mail: najamoto@unicacuca.edu.co

2 Economista, Esp. en Finanzas. Docente Fundación Universitaria Católica Lumen Gentium. Cali, Colombia. e-mail: xmolanot@gmail.com
\end{abstract}

Fecha de recepción: Marzo 2 - $2016 \quad$ Fecha de revisión: Octubre 19 - $2016 \quad$ Fecha de aceptación: Diciembre 12 - 2016

Molano-Tobar NJ, Molano-Tobar DX. Concepción frente al Consumo de Alcohol en jóvenes de la Universidad del Cauca. Univ. Salud. 2016;18(3):532-540. DOI: http://dx.doi.org/10.22267/rus.161803.58

\begin{abstract}
Resumen
Objetivo: Identificar el concepto frente al consumo de alcohol de los jóvenes de la Universidad del Cauca. Materiales y métodos: Cualitativa de diseño etnográfico, a través de observación, entrevista guiada en profundidad con 12 universitarios, 6 hombres y 6 mujeres durante el año 2013 en la ciudad de Popayán-Cauca, con promedios de edad de 17,5 años, que permitió valorar aspectos socio-demográficos y personales sobre el inicio y habituación al alcohol. Resultados: Se identificó que el consumo de alcohol se presenta a temprana edad específicamente durante el periodo escolar, en los últimos niveles del bachillerato y se acentúa en la universidad, ampliándose en esta última la posibilidad de adquirir otras sustancias estimulantes. El consumo de alcohol es debido a las presiones sociales, por el valor simbólico que representa ante sus iguales acerca de la madurez, y la sensación de desinhibición que genera al enfrentarse por primera vez a personas de su otro sexo. Conclusiones: El consumo de alcohol es un medio que emplean los jóvenes para la socialización y la puerta de entrada a nuevas experiencias, por ser una sustancia legal y socialmente aceptada de fácil adquisición y bajo costo, es lo que permite su alto consumo dentro de la población juvenil y universitaria, hecho que amerita generar campañas desde las universidades para la prevención de este hábito.
\end{abstract}

Palabras clave: Alcohol; jóvenes; hábitos; género. (Fuente: DeCS, Bireme).

\begin{abstract}
Objective: To identify the concept against alcohol consumption of young people at the University of Cauca. Materials and methods: An ethnographic qualitative design through observation was performed where 12 university, 6 men and 6 women in 2013 in the city of Popayan Cauca were in-depth guided interviewed with average age of 17.5 years, allowed evaluating sociodemographic and personal aspects about starting and habituation to alcohol. Results: It was identified that alcohol consumption occurs early during the school year specifically, in recent high school levels and is accentuated in college, and extends in the latter the possibility of acquiring other stimulants. Alcohol consumption is due to social pressures, the symbolic value it represents to their peers about maturity, and the feeling of disinhibition generated when confronted for the first time to people of the other sex. Conclusions: Alcohol consumption is a means young people employ for socializing and it represents the gateway to new experiences since it is a legal substance and socially accepted, which is easy to acquire and inexpensive, that allows its high consumption within the youth and university population, a fact that deserves to generate campaigns from universities to prevent this habit.
\end{abstract}


Keywords: Alcohol; young people; habits; gender. (Source: DeCS, Bireme).

\section{Introducción}

La adolescencia es la etapa de la vida que se caracteriza por cambios drásticos y rápidos en el desarrollo físico, mental, emocional y social, que provocan ambivalencias y contradicciones en el proceso de búsqueda del equilibrio consigo mismo y con la sociedad a la que el adolescente desea incorporarse ${ }^{1}$, es por ello que a los adolescentes se les considera como influenciables y maleables, ya que no tienen la experiencia necesaria, para asumir con responsabilidad algunas situaciones de la vida, caso que se presenta con el consumo de alcohol, que es caracterizado como "una de las conductas problemáticas", que conlleva a que el joven actúe de forma inapropiada en las diferentes esferas de su vida y en casos determinados, ocasione accidentes específicamente de tránsito $u$ otros a causa del mismo consumo ${ }^{2}$.

Por lo anterior, el alcohol y el aumento de enfermedades asociadas al mismo consumo, se ha considerado un problema de salud pública que propicia un incremento en "la carga de morbilidad en las Américas"3; situación que amerita ser estudiada, para generar acciones propias con relación a la prevención de este hábito. El consumo de alcohol, lo realiza tanto el alcohólico como el bebedor habitual, sea este moderado o abusivo y el bebedor esporádico, o quien lo hiciera por primera vez, indicando de esta manera que sus efectos se orientan hacia una experiencia determinada que combina dicha y placer ${ }^{4}$.

Han sido diferentes las clasificaciones propuestas, pero la mayoría concuerda con aquel consumo estándar u ocasional, que al final involucra una ingestión superior a cinco tragos ${ }^{3}$.

Investigaciones evidencian que el patrón de consumo a nivel internacional en los jóvenes es hasta "estar ebrios"; dentro de los mismos estudios se ha demostrado una alta prevalencia de ebriedad, así como una tendencia a estar cada vez "más ebrios", término que hoy en día usan como "binge drinking" (consumo episódico excesivo de alcohol) 5 .
La epidemiología internacional como nacional, reportan que el uso ocasional del alcohol, persevera en los jóvenes, generando un interés creciente para los investigadores sobre el tema en el ámbito universitario. El alcohol es considerado como "la droga de inicio en los estudiantes de 12 a 19 años"6.

En Colombia, las estadísticas demuestran que los jóvenes con edades inferiores a los 18 años tienden a consumir más, situación que ha ocasionado que la edad de inicio sea cada vez más temprana a la que se presentaba en años anteriores, donde preliminarmente era de 12 años para los adolescentes colombianos y después en el 2008, fue de 11 años para mujeres y 10 años para los hombres ${ }^{2}$; esto realza la importancia de identificar cuándo comenzaron nuestros jóvenes con la ingesta de alcohol y la causa de ello como uno de los objetivos propuestos para el desarrollo de la investigación. En Estados Unidos el consumo de alcohol y sus complicaciones entre los estudiantes de Educación Superior, ha sido identificado por el gobierno federal, demostrando la gran responsabilidad que hoy en día tienen las mismas instituciones universitarias ${ }^{7}$; por ello la importancia de identificar las motivaciones que tienen los universitarios y como desde la misma institución se puede aportar con planes de promoción de la salud y prevención de la enfermedad.

La incidencia del consumo de alcohol, indica que el aumento de la ingesta de dicha sustancia, se realiza en los tres primeros semestres, debido a que se ven abocados a consumir alcohol y otras sustancias de acuerdo a la cultura universitaria, donde se demuestra ser un joven libre de hacer lo que le plazca y preparado para asumir cualquier reto $^{8}$.

Es notorio, que el consumo se incrementa durante el fin de semana con predilección por la cerveza y los combinados; esto se sustenta en el ámbito colombiano por la disponibilidad y el precio económico, en coherencia con lo descrito, se ha reportado el uso del alcohol no solo se ve 
influenciado por su origen como se pensaba anteriormente, es decir que los jóvenes italianos y franceses consumían solo vino, situación contraria y demostrada con la predilección de la cerveza ${ }^{9}$, revelando que lo importante para los jóvenes en cuanto al consumo de alcohol, es generación de emociones placenteras, productoras de felicidad y que ayuden a vencer sus inhabilidades y hacerle frente a situaciones sociales, debido a los efectos des-inhibitorios del alcohol.

Debido al indudable impacto que el consumo de alcohol genera en los jóvenes, se hace necesario preguntarse sobre la concepción del mismo en los jóvenes de la Universidad del Cauca, realzando la pertinencia social, desde la identificación como el conocimiento que tienen acerca de este aspecto, notando que los jóvenes se encuentran en una fase importante para su proceso de formación hacia una vida adulta con mejor calidad de vida.

Cabe apreciar que este estudio se derivó de la investigación cuantitativa que se realizó con los estudiantes de actividad física formativa, materia transversa del componente socio-humano de la Universidad del Cauca, quienes presentaron una alta frecuencia de consumo de alcohol. Por consiguiente, con la documentación de este estudio se podrán sugerir acciones a favor de adopción de políticas universitarias, construcción de entornos sociales y culturales que fomenten los estilos de vida saludables para estudiantes de la Universidad del Cauca y poder replicar estas acciones en otras universidades de la ciudad y el país, con el fin de generar jóvenes con mayor conciencia hacia su salud.

\section{Materiales y métodos}

\section{Tipo de Estudio}

La investigación fue de metodología cualitativa, utilizando el método etnográfico básico ${ }^{10}$, que permite estudiar lo que en la comunidad ocurre, las distintas interacciones, actividades, valores, ideologías y expectativas de los participantes.

\section{Población}

De 1336 universitarios que contestaron el test AUDIT (conocido en español como el cuestionario de identificación de los trastornos relacionados al consumo de alcohol) ${ }^{11}$, se realizaron reuniones para detectar los estudiantes prestos a participar con alta calificación, bajo los criterios de inclusión; ser estudiante activo de pregrado de la Universidad del Cauca, no presentar historia de alteración mental, asistir continuamente a las reuniones programadas para el proceso investigativo y lo fundamental tener el consentimiento informado firmado, ya sea por ellos como adultos o por su representante legal en el caso de ser menores de edad como manifiesta la declaración de Helsinki ${ }^{12}$ y la resolución 8430 del Ministerio de Salud y Protección Social de Colombia ${ }^{13}$; se evidencio que este proceso fue consentido por 12 participantes distribuidos en 6 mujeres y 6 hombres con edades promedios de 17.5 años (Tabla 1).

\section{Técnicas de Recolección de la información}

La investigación se llevó a cabo durante el año 2013 (enero-diciembre), el trabajo de campo se desarrolló durante los meses de septiembre a diciembre del mismo año.

Para determinar los aspectos sociodemográficos de la población, se suministró una encuesta que ayudó a diferenciar condiciones generales como la edad, estrato socio-económico, procedencia, semestre, facultad y programa al que pertenecen. Respecto al proceso cualitativo como tal, se dividió en dos etapas, la primera donde se concurrió a reuniones o festejos en los ambientes propios de ellos (bares, tabernas, discotecas), de manera individual y su grupo social, aquí el proceso que se utilizó fue el método de observación libre, no sistemática con cuaderno de notas a través de 24 sesiones, que permitió comprender la vinculación de los participantes con su grupo de amigos, las situaciones y experiencias ante el consumo de alcohol; posteriormente se procedió a generar los diferentes encuentros con los 12 participantes en las que se realizaron entrevistas a profundidad registradas en grabaciones de audio y cuaderno 
de notas para llegar a un análisis profundo de sus opiniones y actitudes frente al consumo de alcohol, proceso que generó la determinación de las categorías emergentes.

\section{Análisis de la información}

El análisis se realizó bajo el método de comparación constante en tres fases: acercamiento, donde se realizó una aproximación a los jóvenes participantes; focalización, empezando con las grabaciones y entrevistas en profundidad, hasta la codificación y categorización de la información de manera simple o abierta, axial y selectiva, conforme a los patrones y tendencias que se descubrieron tras la lectura y el análisis constante de los mismos; de este modo se identificó las distintas piezas de información de acuerdo con criterios de ordenamiento como agrupamiento y finalmente, profundización, que consistió en alcanzar la saturación de las categorías empíricas y su descripción. Se codificaron los relatos teniendo en cuenta la identificación de las personas con "María" si eran mujer y con el nombre de "Hernán" para el caso de los hombres y el número del relato al que pertenecía se codifico bajo la letra $\mathrm{R}$.

\section{Consideraciones éticas}

Se tuvo en cuenta lo dispuesto en la declaración de Helsinki $1975^{12}$ y la Resolución 8430 de 1993 del Ministerio de Salud y Protección Social de Colombia para investigación con personas ${ }^{13}$, se presentó el proyecto a los jóvenes y en los casos de ser menores de edad a los representantes legales y una vez explicado cómo comprendido, se diligenció el consentimiento informado para mayores de edad y asentamiento en caso de menores de edad, donde se garantiza la protección a la intimidad y confidencialidad de los datos suministrados como de las entrevistas, considerando el estudio con riesgo mínimo de lesión para los participantes.

\section{Resultados}

\section{Características Socio-demográficas}

Con los datos de la encuesta realizada previa al análisis cualitativo, se evidenció que la procedencia de los jóvenes estudiantes es de la ciudad de Popayán-Cauca y pertenecientes en general al estrato socio-económico 3; (Tabla 1), de igual manera, se observó que los participantes pertenecen principalmente a la Facultad de Ciencias Naturales, Exactas y de la Educación (25\%), de Ingeniería Civil y Ciencias Agropecuarias (16,7\%). Los participantes fueron clasificados en su totalidad como consumidores de fin de semana y el tipo de alcohol que consumen con mayor frecuencia es la cerveza (57\%), seguido del aguardiente con un (22\%).

El promedio de edad de la población estudiada correspondió a 17,5 años y la edad promedio de inicio del hábito de consumo de alcohol es de 13,5 años, se observa un comienzo temprano en los hombres (12 años) que de las mujeres (15 años). Al realizar el análisis, se obtuvo las siguientes categorías (Tabla 1).

Tabla 1. Aspectos socio-demográficos

\begin{tabular}{lrrr}
\hline & \multicolumn{1}{c}{$\begin{array}{c}\text { Estrato } \\
\text { socioeconómico }\end{array}$} & Edad & Semestre \\
\hline Media & 3,00 & 17,50 & 3,58 \\
Desviación & 1,04 & 1,78 & 1,62 \\
estándar & & & \\
Mínimo & 2 & 15 & 2 \\
Máximo & 5 & 20 & 7 \\
\hline
\end{tabular}

\section{Beber, algo natural sinónimo de estar listo a madurar}

Es indudable que los jóvenes consideran la ingesta de alcohol como algo normal de su proceso de maduración y ese algo que deben enfrentar en cualquier circunstancia, es considerado como un desafío de sus iguales, además consideran que es permitido, debido a que desde sus casas se observa este hábito "pues mira, cuando empecé a rumbear comencé a tomar, eso está ligado fiesta-trago, es algo de nuestra cultura colombiana, que vemos en nuestras casas desde pequeños, por cualquier cosa se toma, si ganamos, si perdemos" (R2Hernán3); "realmente, yo tuve mis primeros encuentros con el trago en las fiestas de quinces de mis amigas, que fue cuando yo ya estaba lista para ser más adulta y menos niña" (R2María2). 


\section{Con un trago me enfrento al mundo}

La sensación que experimentan nuestros jóvenes con la ingesta de alcohol, se refleja en la liberación, en la trasformación del tímido a volverse audaz, capaz de vencer el mundo y sus problemas. Se presenta también una modalidad de salir ganador ante sus iguales como la sociedad, ir más allá de los límites, situaciones que sin el alcohol no se lo permitirían, "es como te digo, dos tragos liberan, tres desinhibe y cinco o seis entorpece, por eso cuando sé que es lo que quiero voy por ello, y cuando estoy en una fiesta, detrás de una nena no me los tomo todos, me mido y asígano" (R3Hernán3); "como dicen mis amigas soy la más, más y es que con un par de traguitos no hay tipo que me resista, se lo que quiero y a ello llego" (R2María4).

\section{Mis inicios más por presión social que por convicción}

Las fiestas y reuniones se han convertido en el foco de ingreso para acceder a la ingesta de alcohol, donde se generan directa o indirectamente presiones sociales de acercamiento con otros, son los puntos de movilización, de encuentro, de información y compromiso. "yo me había hecho el propósito de nunca tomar, (hhh) pues con la experiencia de mi papa con el trago, me daba pereza, pero cuando uno comienza a ir a fiestas cambia la cosa, los amigos le invitan un chorrito y uno lo toma más por calmar los nervios y las ansias" (R2Hernán4); "yo no bebía, sino que comencé a salir con el parche de mi novio y como ellos son más recorridos pues me decían que yo si no tomaba, pues no era parte de la gente cool, y como me gustaba tanto salir con ellos y el ambiente que se presentaba, pues lo hacía" (R2María1).

\section{Con el licor, más sensual y más sexual}

Es evidente como el alcohol se ha convertido en un referente que desinhibe a los jóvenes y en su representación social, es sinónimo de empoderamiento y atracción por parte del otro sexo, convirtiendo al joven o la joven como un ser sensual, que responde sexualmente ante situaciones que lo requieran. "recuerdo mucho cuando me gustaba una chica, y como varios de nosotros andábamos atrás de ella, pero en una fiesta se me hizo, ya que cuando llego yo me había entonado y me lance a dedicarle y cantarle una canción, eso fue lo que a ella le gusto y de ahí en adelante me la charle" (R3Hernán1); "yo no era muy sociable como mis amigas, me daba pena con los chicos, hasta que un día di rienda suelta a mi yo escondido y me puse a bailar, me dijeron que lo hacía muy sensual, obvio después de tomarme unos traguitos y de ahí las cosas fluyeron tenía más admiradores" (R2María5). De la misma manera la forma como asumen la sensualidad y sexualidad se relaciona con el género, lo hombres se ven sexuales mientras que las chicas se asumen sensuales.

\section{El alcohol un des inhibidor y liberador fácil de encontrar}

La sociedad fomenta el uso e incluso el abuso del alcohol, debido a que es una sustancia estimulante legalmente aceptada, y aunque se han planteado numerosas propuesta para regular su consumo en el país, falta más conciencia por parte de los vendedores como de los consumidores; la representación social que ellos manifiestan son conceptos positivos acerca de lo que obtienen que las consecuencias fruto de su abuso. "a donde tu vayas encuentras bebidas alcohólicas, tanto es así que cerca a la universidad, están las cafeterías donde tomarse una cerveza, que te da pie para conocer a más pelaas y después salir a parrandear" (R3María6); "es más bravo que te vean con un porro a que te vean con una cerveza, tanto así que es más probable que uno puede invitar a un profe a tomarse una cerveza que a fumarse otra cosa" (R3Hernán6).

\section{Popayán y su rumba}

Según las encuestas del DANE del año 2005, Popayán es una de las ciudades con mayor índice de desempleo en el país, hecho que ha generado que los ciudadanos busquen fuentes de empleo para mitigar esta situación, una de las opciones son la creación y apertura de bares y "rumbeaderos", que vinculan a la vida nocturna a los jóvenes, de la misma manera no hay evidencia de un control por parte de los establecimientos, como lo mencionan los jóvenes "mi parche es el más caliente, cada fin de semana comenzando desde el jueves, salimos a rumbear y pasarla bueno, y con la cantidad de sitios que ahora hay pues no la pasamos mejor" (R5Hernán6); "mira no es para 
negar que el viernes es de desbarate, con mis amigas nos ponemos la percha y a rumbear, Popayán lejos de ser una ciudad universitaria es más infierno del desorden" (R6María4).

La UNESCO el 30 de septiembre de 2009, reconoció a la Semana Santa de Popayán, como Patrimonio Cultural Inmaterial de la Humanidad, Fiesta religiosa que se caracteriza por la procesión de imágenes que reproducen la pasión, muerte y resurrección de Jesucristo; por las calles del centro de la ciudad, se realizan diariamente la procesión, donde se representa el orden social de la ciudad, desfilando las autoridades morales, religiosas, políticas, educativas y militares, semana que llama a los jóvenes más al desorden y a la rumba que a la reflexión y unión, "siiii, Popayán es reconocida por la parranda santa, es la semana en la cual reconozco, más bebo del año, además me da pie a conocer gente de otras partes del país" (R5Hernán5); "es full esa semana, no hay clase y Popayán se convierte en el mayor rumbeadero con más sitios de diversión que en otra fecha, tanto dentro como a los alrededores y como dice un grafiti paredes blancas almas negras, porque durante la procesión es la rezadera y calma, después a desordenarse, y como viene tanta gente uno ve de todo y me refiero a todo" (R6María2).

\section{La salud: Para pensarlo en el futuro}

Uno de los ítems que más se presentó con frecuencia en las conversaciones, eran las relacionadas con los aspectos de la salud, evidenciando que no es un tema de preocupación en la actualidad para sus vidas y lo que se busca en el momento es disfrutar y pensar después, "uno sabe por los medios que hay muchas muertes por pelaos que manejan de nuestra edad que consumen trago, pero uno que es un vaciao se la pasa en bus y buseta eso no le pasara, y en relación a enfermedades pues ahora estamos jóvenes y hay que disfrutar" (R3Hernán5);"yo sé por lo que he estudiado que el trago es un causante de muchas enfermedades, pero si uno se regula en el consumo se puede manejar, igual habrá tiempo y Dios dará los medios económicos para solucionar los inconvenientes" (R4María5).

\section{Discusión}

En la investigación se demostró que los estudiantes universitarios presentan un alto grado de consumo de alcohol, ante ello estudios sugieren la "evidencia que el alcohol es la fuente de entrada", hacia el consumo de nuevas sustancia psicoactivas ${ }^{2}$.

La población universitaria presentó una edad promedio de inicio de consumo a los 13,5 años; para el caso de los hombres se encontró que empiezan a los 12 años y a una edad posterior las mujeres (15 años), en estudios similares, "la edad temprana de inicio del uso del alcohol era de 12 años en los adolescentes colombianos, ahora es de 11 para las mujeres y 10 para los hombres"2; este hecho demuestra cómo la ingesta de alcohol es explicada desde "la valoración social y simbólica que asocian a la virilidad en el hombre, demostrando que el acto de beber prueba la masculinidad, que exacerba el machismo, que refrenda el carácter mujeriego como aventurero" 14 y a esto se le suman los motivos hacia la permisividad de los padres y el tipo de familia al que pertenecen.

Por otro lado, se logró demostrar que en la mayoría de los participantes presentaban cierto grado de influencia familiar en la adquisición de este hábito, ya que lo observan desde pequeños, estudios en poblaciones indígenas de Colombia demuestran que "A los niños se le da plátano en polvo bien molidito en colada y chicha que no es amarga, es dulce"15, la chicha "bebidas con distintos grados de fermentación a base de maíz o caña de azúcar" y la cual también se suministra en diferentes festejos propios de las comunidades ${ }^{16}$.

Se evidenció que los universitarios tienen disponibilidad para el consumo de bebidas alcohólicas y provienen en su mayoría de un estrato socio-económico medio para la ciudad de Popayán, situación que algunos estudios consideran un factor de riesgo, debido a que a mayor nivel adquisitivo, mayores relaciones con el incremento en el consumo de alcohol y otras substancias, pues jóvenes que "reciben cantidades elevadas de dinero por parte de sus padres" acentúan la posibilidad de accesibilidad y 
a la vez "un mayor involucramiento en actividades de alto riesgo para el consumo"7; hecho que amerita ser estudiado desde la dimensión subjetiva de ser padre hoy en día.

Por otra parte se evidencia a nivel intra-grupal, que los hombres de estrato socio-económico bajo, tienden a aumentar su ingesta por diferentes situaciones como son las asociadas a la masculinidad o por presión social, aspecto relacionado en algunas investigaciones donde se menciona que los hombres asumen diferentes posiciones debido a "una fuerte necesidad de ser aceptados y obtener un reconocimiento social" 17 convirtiéndose el alcohol en un "instrumento social para negociar un estatus y roles" 18 .

Otros estudios ${ }^{19}$ corroboran la percepción que presentan los jóvenes frente al inicio del consumo de alcohol, explicada por las ganas de madurar, de sentirse mayores y pertenecer a un grupo, ante ello Manrique et al., señala que "la maduración temprana y algunos aspectos derivados de la pubertad funcionan como factores de riesgo para el consumo de alcohol" y pueden ser un detonante para involucrarse en el uso y abuso de otras sustancias más complejas y de mayor riesgo para su salud ${ }^{20}$.

Desde este estudio, se pudo identificar que otro factor de riesgo para el consumo, es la alta disponibilidad del producto, evidenciándose en los establecimientos que rodean a las universidades y más presentes en la ciudad de Popayán, ya que el precio por el cual se consigue una cerveza es muy similar a la de una bebida gaseosa, esto es sustentado y corroborado por un estudio de Acosta et al., que menciona "la bebida más consumida es la cerveza, en especial los fines de semana"5.

Según la investigación de Gil, el consumo está influenciado por la representación que tiene la cerveza para la población en general, consideran que "la cerveza cumple con una función comunitaria, que acompaña los intercambios comunicacionales y ayuda a construir una atmósfera festiva y amistosa"21; factores explicativos para Sur América, que por ser comunidades con descendencia indígena, que en las tradiciones y celebraciones "abunda la música ancestral, los bizcochuelos, la comida, la chicha y el aguardiente en la iglesia y en cada hogar" 22 en contraste con Europea en países como Francia e Italia cuya producción y consumo es en torno al vino.

La ingesta de alcohol es frecuente en reuniones y eventos sociales, tal manifestación es común en investigaciones que denotan que esta conducta de consumo se enmarca en el contexto de reuniones sociales como de eventos especiales, y se evidencia que quienes ofrecen las bebidas son desde los mismos familiares hasta los amigos más cercanos $^{23}$. Tal situación es corroborada y se explica en gran parte desde el contexto social que Colombia presenta, el cual "es un país donde el alcohol hace parte de la canasta familiar, convirtiéndose en un estilo de vida, que legalmente es aceptado e inclusive las empresas productoras patrocinan y financian numerosos espectáculos recreativo"4. Este hecho presenta una realidad, en la cual el joven se ve inmerso y muestra una sociedad que vende este imaginario como un hábito natural y fácil de asimilar.

La apreciación que este estudio demostró, es que los jóvenes tienen la capacidad de enfrentar cualquier adversidad, sustentado desde Londoño et al. ${ }^{24}$, quienes señalan cómo el alcohol es un facilitador social y genera una expectativa de reducción de la tensión psicológica, así mismo desde esta investigación se evidenció como los jóvenes observan que el consumo de alcohol es un medio de desinhibirse, lo que les da la imagen de fuerza y protección, la función activa de acercamiento a la mujer y desempeñarse mejor sexualmente ${ }^{14}$, con la suposición o pensamiento de responder mejor a la tarea de ser "un verdadero macho", por lo tanto el joven toma para afrontar sus miedos y tabúes, para buscar la seguridad y desinhibición deseadas en el intercambio sexual.

Desde la antigüedad la mujer fue considerada para labores domésticas y cuidado de los hijos, pero hoy en día ese rol se ha ampliado, permitiendo que la mujer acceda a otras dinámicas que antes eran exclusivamente sólo para los hombres, pero con relación al consumo 
de alcohol, este ha sido reservado y con múltiples prejuicios sociales; en este sentido se encuentra que investigaciones de Martínez ${ }^{25}$, indican que las edades pico de consumo en las mujeres esta entre los 25 a 29 años, para este estudio se evidenció que la tendencia se ha reducido y está presente en edades universitarias, esto debido a las presiones sociales (grupo-novio), como a los diferentes roles que hoy en día asume la mujer. Otros autores concuerdan con estas evidencian y relacionan el aumento de consumo con el control de afrontamientos, la autoevaluación, la autoestima, la afectividad, los conocimientos, la afectividad, el estilo de vida y la fortaleza social hacen que la mujer incrementen su adicción ${ }^{26,27}$.

Al identificar las relaciones que tienen los jóvenes sobre el consumo de alcohol con la salud, se evidencia que aunque hay un conocimiento pobre, obtenido por los medios de comunicación especialmente, no es una preocupación actual o de su condición y "esto ha llevado a que se consuma cada vez más" 28 . Por otra parte se presenta en el estudio de Góngora y Leyva ${ }^{14}$, el cambio de referentes y son sustentadas por las condiciones "tanto macro como microestructurales que cruzan todos los ámbitos de la vida" que ha incorporado a la mujer al mercado de trabajo. De la misma manera en la población indígena se ha cambiado tal concepción indicando que: "la niña abandona la casita y arroja al rio mochilas que ha tejido, sin mirar atrás botando la pereza y recibiendo ánimo para trabajar. [...] la niña prepara los alimentos, reparte la comida, la chicha a todos los asistentes, integrándose como nueva mujer en el ámbito social de la comunidad"29.

Otro parámetro que sustenta el incremento de ingesta de alcohol en las estudiantes UniCaucanas, es que la mayoría provienen de familias separadas o divorciadas, situación que para Becoña et al. ${ }^{30}$, puede generar un condicionamiento y tendencia al alcohol, al igual que la predisposición por factores como la vulnerabilidad biológica y psicosocial asociados a "grado significativamente alto de depresión, pobreza en su autoestima, afectación en su autocontrol con sentimientos y actos agresivo" 25 .
Los esfuerzos que se publican en torno a los efectos negativos del alcohol sobre la salud, son pocos al comparar el notorio impacto de la influencia que la publicidad ejerce hacia el consumo, y a pesar de los programas de prevención que se han puesto en marcha, "la prevalencia de consumo aumenta, pasando en el 1997 de $64 \%$ al 62,3\% en el 2011, y el mayor consumo y publicidad "se concentra en la cerveza y las bebidas de alta graduación”31.

Para los investigadores es necesario generar otros estudios que problematicen la relación del consumo de alcohol con el género, como también la incidencia de la vulnerabilidad familiar hacia este problema social.

\section{Conclusiones}

Se pudo identificar que los adolescentes asumen el consumo de alcohol desde temprana edad, y muchas veces con la aprobación de su familia y amigos.

El consumo de alcohol se presenta debido a las presiones sociales, por el valor simbólico que representa ante sus iguales sobre madurez y la sensación de ser adultos.

Es evidente como la ingesta de alcohol se ha incrementado en el género femenino, para lo que se hace necesario un trabajo sobre su autoestima y mejoramiento de los estilos de vida, como de las consecuencias sobre su salud.

Los jóvenes identifican las complicaciones en la salud que trae consigo la ingesta de alcohol, pero no consideran que puedan afectarles en este momento.

Es necesario el diseño de programas integrales para la prevención del consumo de alcohol, desde el escenario familiar, escolar y comunitario tendiente a minimizar la ingesta y prevenir la utilización o combinación con otras sustancias.

La publicidad es un medio que influye hacia el consumo, por el múltiple bombardeo que ejerce 
en diferentes medios y vincula cada vez más a la población menor de edad.

\section{Agradecimientos}

A todos y cada uno de los colaboradores quienes por su libertad en la expresión y honestidad ayudaron a responder muchas preguntas. De igual manera a todos nuestros compañeros del Departamento de Educación física de la Universidad del Cauca, que desde su práctica colaborativa apoyaron con sus comentarios y apreciaciones.

Conflicto de intereses: Ninguno declarado por los autores.

\section{Referencias}

1. Rodrigo MJ, Máiquez ML, García M, Mendoza R, Rubio A, Martínez A, et al. Relaciones padres-hijos y estilos de vida en la adolescencia. Psicothema. 2004;16:203-10.

2. Trujillo A, Flórez IA. Consumo de alcohol en los adolescentes de Chía y su percepción del consumo y de la permisividad parental frente al uso de sustancias. Rev Colomb Psicol. 2013;22:41-57.

3. Salcedo A, Palacios X, Espinosa AF. Consumo de alcohol en jóvenes universitarios. Av en Psicol Latinoam. 2011;29(1):77-97.

4. Camacho I. Consumo de alcohol en universitarios: Relación funcional con los factores sociodemográficos, las expectativas y la ansiedad social. Acta Colomb Psicol. 2005;13:91-119.

5. Acosta L, Fernández AR, Pillon SC. Factores sociales para el uso de alcohol en adolescentes y jóvenes. Rev LatinoAmericana Enfermegem. 2011;19:771-81.

6. Herrera-Vázquez M, Wagner F a., Velasco-Mondragón E, Borges G, Lazcano-Ponce E. Inicio en el consumo de alcohol y tabaco y transición a otras drogas en estudiantes de Morelos, México. Salud Publica Mex. 2004;46(2):132-40.

7. Díaz A, Díaz R, Hernández C a, Narro J, Fernández H, Solís C. Prevalencia del consumo riesgoso y dañino de alcohol y factores de riesgo en estudiantes universitarios de primer ingreso. Salud Ment. 2008;31(4):271-82.

8. Londoño C, Valencia SC, Sanchez L, León V. Diseño del Cuestionario resistencia a la presión de grupo en el consumo de alcohol (CRPG). Suma Psicol. 2007;14:27188.

9. Salazar IC, Arrivillaga M. El consumo de alcohol, tabaco y otras drogas, como parte del estilo de vida de jóvenes universitarios. Rev Colomb Psicol. 2008;13:74-89.

10. Murcia N, Jaramillo LG. Investigación cualitativa. "La complementariedad etnografica". Una guia para abordar estudios sociales. Armenia. Ed Kinesis; 2000: 1-228.

11. Castañeda J, Chaves L. Relaciones entre estilos de personalidad y tipos de consumo de alcohol. Pensam Psicol. 2013;11:55-69.

12. Medica A. Declaracion de Helsinki. Principios éticos para las investigaciones médicas en seres humanos. Tokio-
Japón: Asociación Medica Mundial; 2008: 5.

13. Colombia. Ministerio de Salud y Protección Social. Resolución 8430 de 1993. Bogota: 1993: 1-12.

14. Góngora J, Leyva M. El alcoholismo desde la perspectiva de género. El Cotid. 2005;20:84-91.

15. Mantilla $\mathrm{B}$, Oviedo $\mathrm{M}$ del $\mathrm{P}$, Hernández A, García NZ. Educación para la salud una expriencia con población indigena del Departamento de Chocó. Rev Hacia la Promoc la Salud. 2013;18:96-109.

16. Rosique J, Restrepo MT, Manjarrés LM, Gálvez A, Santa J. Estado nutricional y habitos alimentarios en indigenas Embera de Colombia. Rev Chil Nutr. 2010;37:270-80.

17. Lema LF, Varela MT, Duarte C, Bonilla M V. Influencia familiar y social en el consumo de alcohol en jóvenes universitarios. Rev Fac Nac Salud Pubñica. 2011;29:26471.

18. Vázquez V, Roberto G. Masculinidad hegemonica, violencia y consumo de alcohol en el medio universitario. Rev Mex Investig Educ. 2009;14:701-19.

19. Pimentel CE, Gomes T, Vera JÁ, Barroca G. Actitud, Intencion y uso de bebidas alcoholicas. Acta Investig Psicol. 2014;4(1):1356-69.

20. Manrique-Abril FG, Ospina JM, Garcia-Ubaque JC. Consumo de alcohol y tabaco en escolares y adolescentes de Tunja, Colombia, 2009. Rev Salud Publica. 2011;13(1):89-101.

21. Gil J. Te sigo a todas partes. Pasión y aguante en una hinchada de fútbol de un club del interior. Intersecc en Antropol. 2006;7:333-48.

22. Peña JA. En Las coces del Pishimisak: Apuntes etnográficos sobre el tiempo Misak. Maguaré. 2009;23:343-69.

23. Novoa-Gómez MM, Barreto I, Silva LM. Consumo de cigarrillo y prácticas culturales en contextos universitarios. Rev Latinoam Psicol. 2012;44(1):97110.

24. Londoño C, García W, Valencia S, Vinaccia S. Expectativas frente al consumo de alcohol en jóvenes universitarios colombianos. An Psicol. 2005;21(2001):259-67.

25. Martínez A. Alcoholismo femenino: Problemática social. Rev Estud Genero La Vent. 2002;2:78-95.

26. Pilav A, Rudi A, Brankovi S, Djido V. Perception of health risks among adolescents due to consumption of cigarettes, alcohol and psychoactive substances in the Federation of Bosnia and Herzegovina. Public Health. 2015;9:1-7.

27. Ramos M. La mujer y el alcoholismo en México en el siglo XIX. Salud Ment. 2001;24:24-8.

28. Medina-Mora ME, Real T, Villatoro J, Natera G. Las drogas y la salud pública: ¿hacia dónde vamos? Salud Publica Mex. 2013;55(1):67-73.

29. Tombe A julia, Morales MA, Tunubala S. Las expresiones de afectividad en la familia Misak en el espacio del Nachak-fogon. Journal of Chemical Information and Modeling. 2013.

30. Becoña E, Miguez MC, Lopez A, Vazquez M jose, Lorenzo M carmen. Resiliencia y consumo de alcohol en jóvenes. Salud Drogas. 2006;6:89-111.

31. López-Sánchez C, García JA, Gázquez M. Influencia de la comunicación publicitaria en el consumo de alcohol. Hist y Comun Soc. 2013;18:639-55. 